\title{
Penile cancer brachytherapy HDR mould technique used at the Holycross Cancer Center
}

\author{
Robert Matys, MD, Iwona Kubicka-Mendak, MD, Jarosław Łyczek, MD, PhD, Piotr Pawłowski, MSc, Iwona Stawiarska, MD, \\ Joanna Miedzinska, MSc, Paweł Banatkiewicz, MD, Aldona Łaskawska-Wiatr, MD, Justyna Wittych, MSc \\ Holycross Cancer Center, Kielce, Poland
}

\begin{abstract}
The aim of this pictorial essay is to present the mould based HDR brachytherapy technique used at the Holycross Cancer Center for penile cancer patients. We use images to describe this method step by step.

Key words: penile cancer, HDR brachytherapy, mould.

\section{Case report}

Penile carcinoma is a rare type of cancer. In Poland it occurs in about five cases in a million men. The exact cause is not known (as in other cancer types). The most suspected is HPV infection and bad hygiene status; early circumcision may prevent development of this tumour. The most common treatment for patients is surgery. It is effective but often connected with loss of the penis or its functionality. Brachytherapy has been used as an alternative, organ-preserving treatment option for several decades. Results achieved with this method were comparable to surgery. Brachytherapy initially was used as LDR and then as PDR or HDR technique [1-12].

There are two types of application and treatment: 1) interstitial, requiring anaesthesia and short hospitalization; 2) mould technique based on individual contact applicator. In our department we decided to choose the second one for patients' convenience, the possibility to introduce 3D CT based planning and potentially better cosmetic effect using HDR sources (more homogeneous irradiation). This method allows treatment to be applied on an outpatient basis. We used the following treatment schema: Total dose: 15 fractions, 3 Gy per fraction; 5 days/week on 19 consecutive days. We present our whole procedure from qualification through preparing the applicator, irradiation to the early results.

Conclusions: Mould based HDR brachytherapy used at the Holycross Cancer Center for penile cancer is a very convenient, repeatable method. It allows preservation of the treated organ with full functionality. The procedure can be done on an outpatient basis. Use of this type of HDR brachytherapy is safe (afterloading) and precise (CT based planning). Efficacy could not be assessed due to the small group $(n=5)$ of patients but early results are promising.

\section{References}

1. De Crevoisier R, Slimane K, Sanfilippo N et al. Long-Term Results of Brachytherapy for Carcinoma of the Penis Confined to the Glans (N- or Nx). Int J Radiat Oncol Biol Phys 2009; 74: 1150-1156.

2. Crook J, Grimard L, Tsihlias J et al. Interstitial brachytherapy for penile cancer: An alternative to amputation. J Urol 2002; 167: 506-511.

3. Mazeron JJ, Langlois D, Lobo PA et al. Interstitial radiation therapy for carcinoma of the penis using iridium 192 wires: The Henri Mondor experience (1970-1979). Int J Radiat Oncol Biol Phys 1984; 10: 1891-1895.

4. Chaudhary AJ, Ghosh S, Bhalavat RL et al. Interstitial brachytherapy in carcinoma of the penis. Strahlenther Onkol 1999; 175: 17-20.

5. Petera J, Odrazka K, Zouhar M et al. High-dose-rate interstitial brachytherapy for the treatment of penile carcinoma. Strahlenther Onkol 2004; 180: 123-125.

6. Crook JM, Jezioranski J, Grimard L et al. Penile brachytherapy: Results for 49 patients. Int J Radiat Oncol Biol Phys 2005; 62: 460-467.

7. Rozan R, Albuisson E, Giraud B et al. Interstitial brachytherapy for penile carcinoma: A multicentric survey (259 patients). Radiother Oncol 1995; 36: 83-93.

8. Akimoto T, Mitsuhashi N, Takahashi I et al. Brachytherapy for penile cancer using silicon mold. Oncology 1997; 54: 23-27.

9. Petera J, Sirák I, Kašaová L et al. High-dose rate brachytherapy in the treatment of penile carcinoma - first experience. Brachytherapy 2011; 10: 136-140.

10. Crook J. Radiation therapy for cancer of the penis. Urol Clin North Am 2010; 37: 435-443.

11. Crook J, Jezioranski J, Cygler JE. Penile brachytherapy: technical aspects and postimplant issues. Brachytherapy 2010; 9: 151-158.

12. Langsenlehner $\mathrm{T}$, Mayer R, Quehenberger $\mathrm{F}$ et al. The role of radiation therapy after incomplete resection of penile cancer. Strahlenther Onkol 2008; 184: 359-363. 


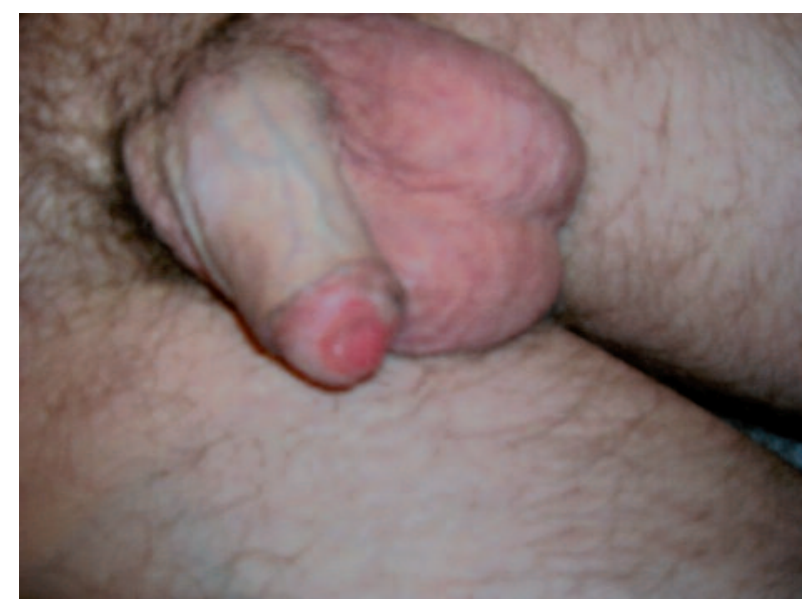

Fig. 1. I. Qualification: Patients T1-T2, superficial or infiltrative lesions are suitable. Eventually patients with more advanced disease who definitely refuse other treatment options

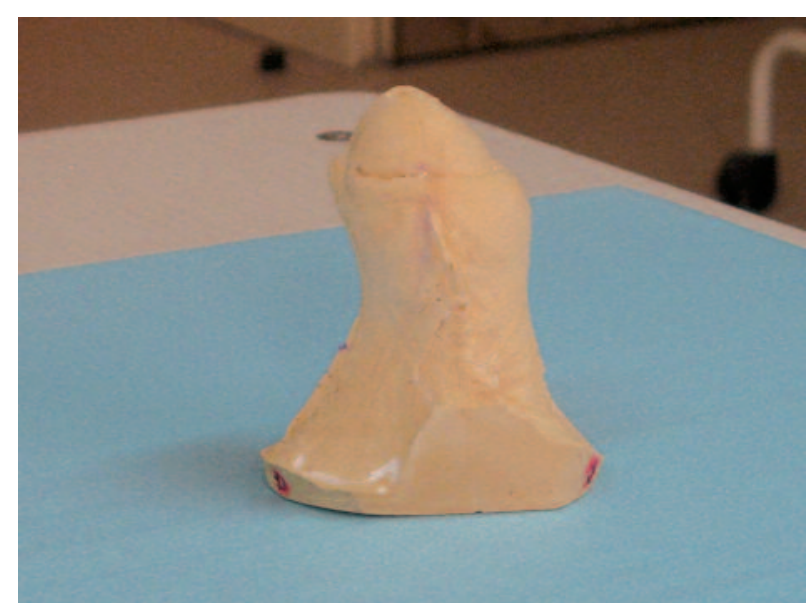

Fig. 3. II. Preparing: Individual mould based applicator with catheters placed around penis

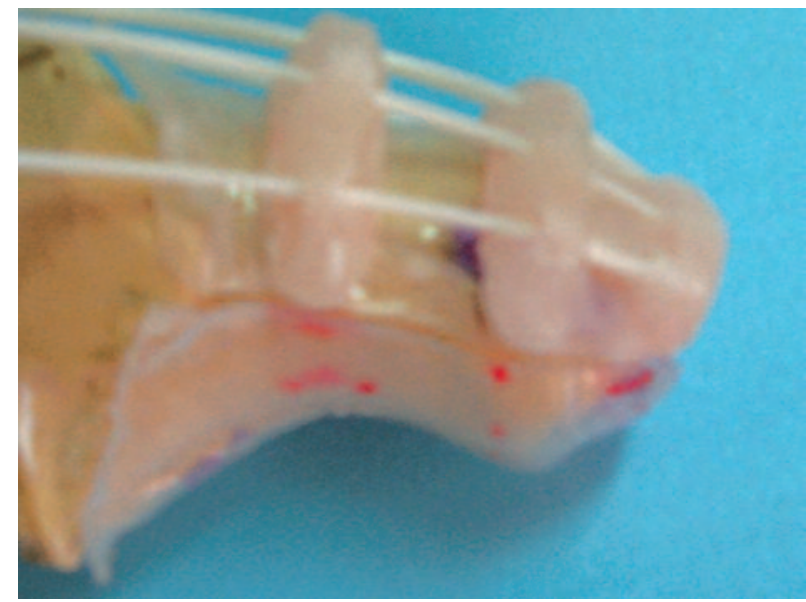

Fig. 5. II. Preparing: Individual mould based applicator with catheters placed around penis

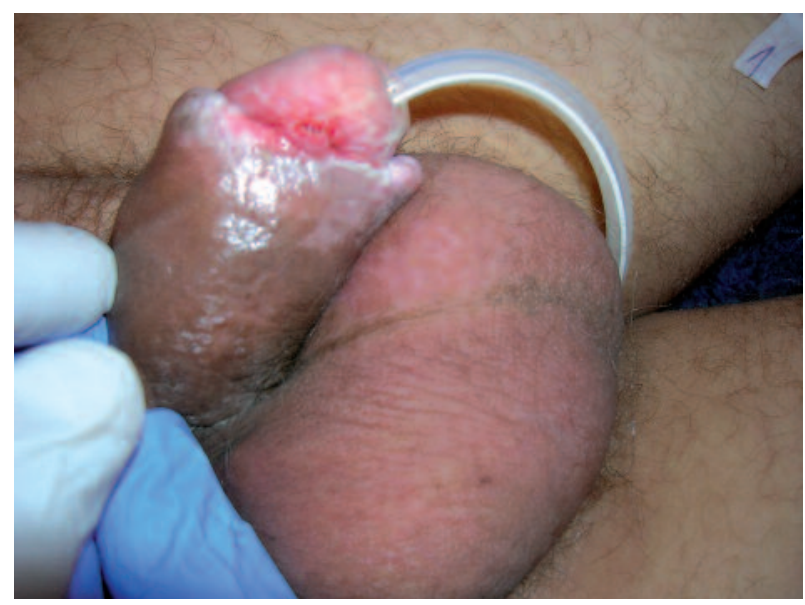

Fig. 2. I. Qualification: Patients T1-T2, superficial or infiltrative lesions are suitable. Eventually patients with more advanced disease who definitely refuse other treatment options

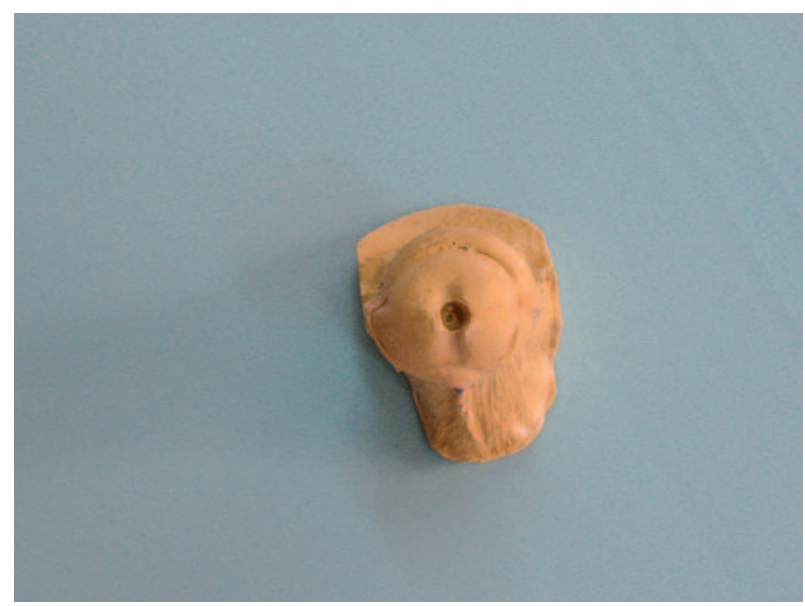

Fig. 4. II. Preparing: Individual mould based applicator with catheters placed around penis

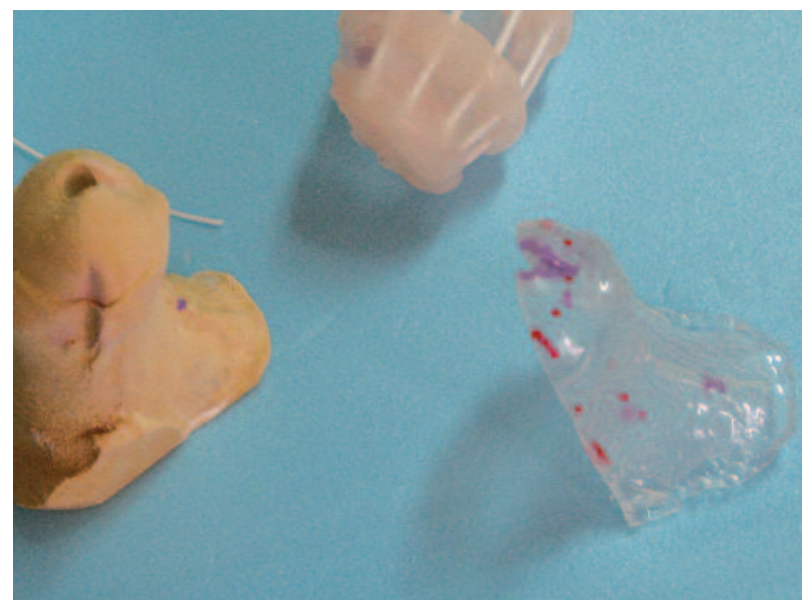

Fig. 6. II. Preparing: Individual mould based applicator with catheters placed around penis 


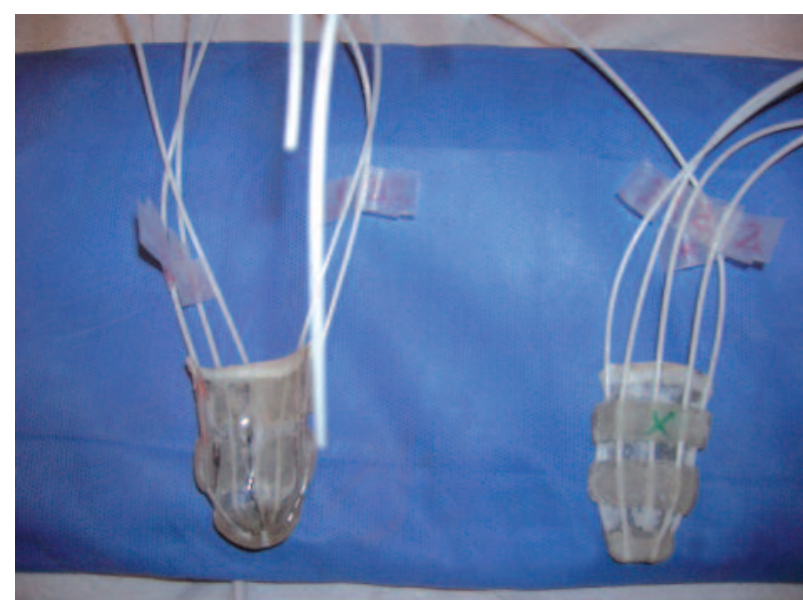

Fig. 7. II. Preparing: Individual mould based applicator with catheters placed around penis

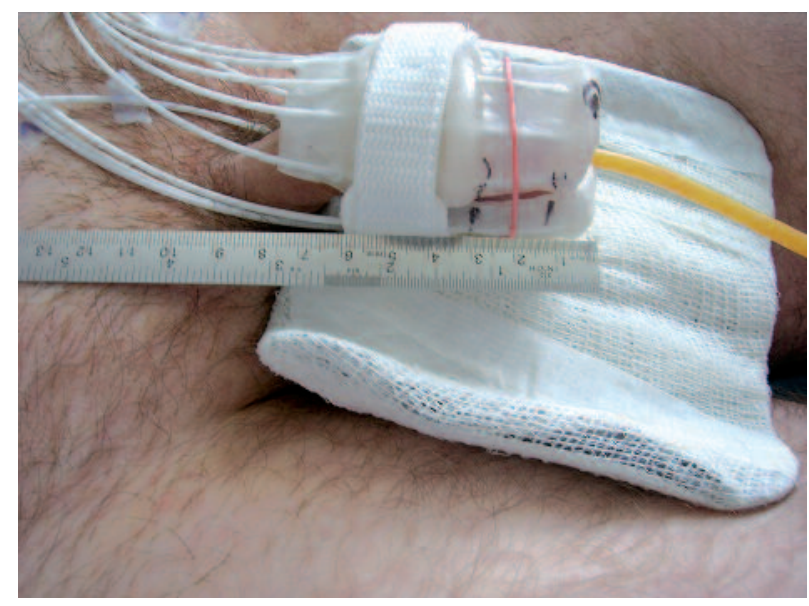

Fig. 9. II. Preparing: Individual mould based applicator with catheters placed around penis

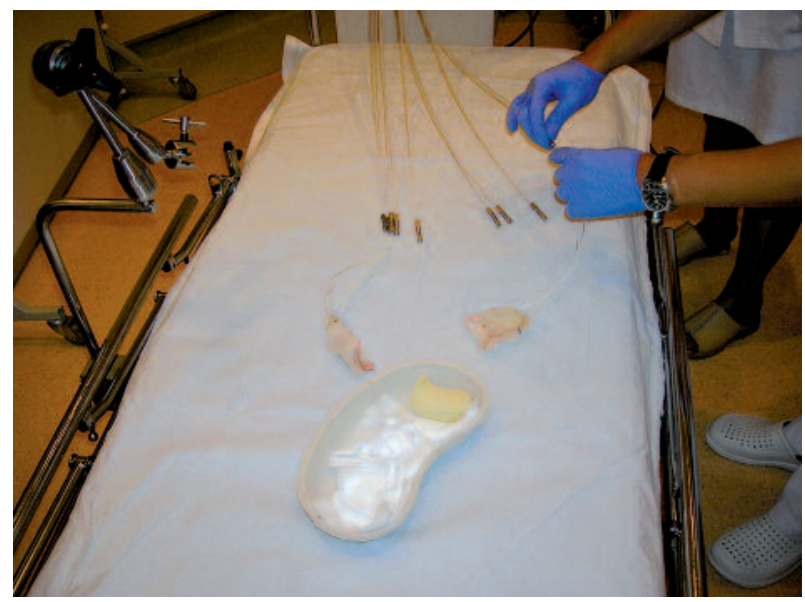

Fig. 13. IV. Therapy session: Preparing applicator, placing organ into it, Mosfet dosimetry, scrotum shielding, irradiation

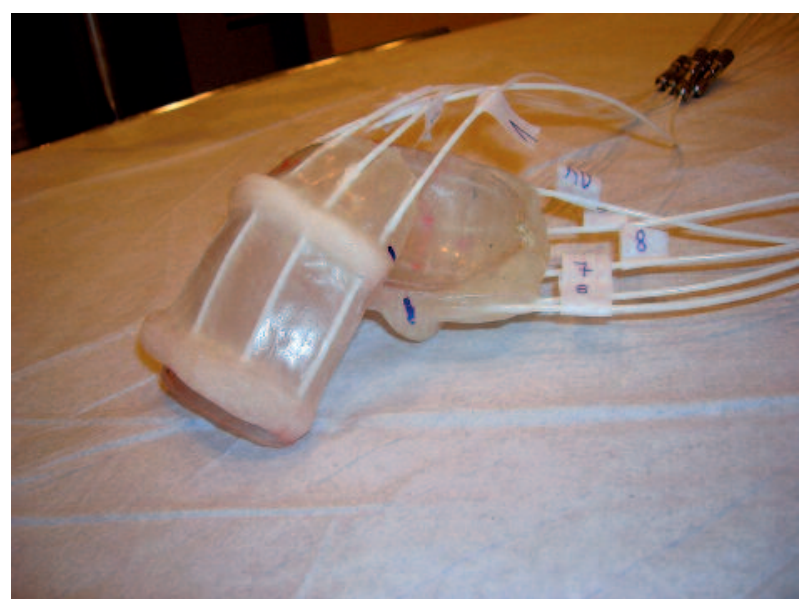

Fig. 8. II. Preparing: Individual mould based applicator with catheters placed around penis

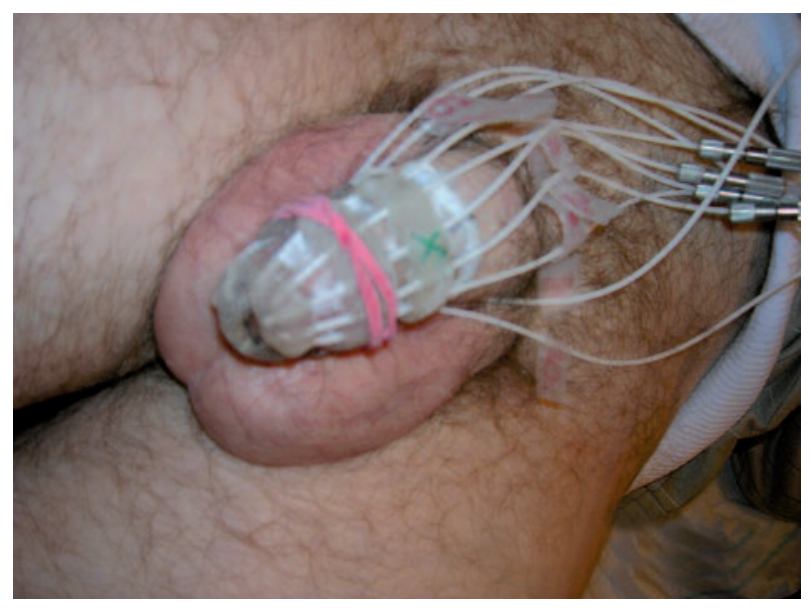

Fig. 10. II. Preparing: Individual mould based applicator with catheters placed around penis

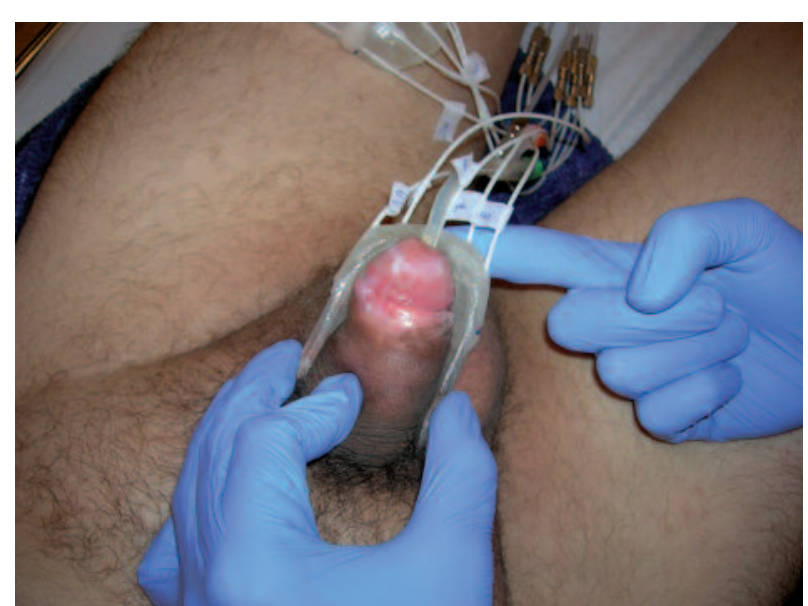

Fig. 14. IV. Therapy session: Preparing applicator, placing organ into it, Mosfet dosimetry, scrotum shielding, irradiation 


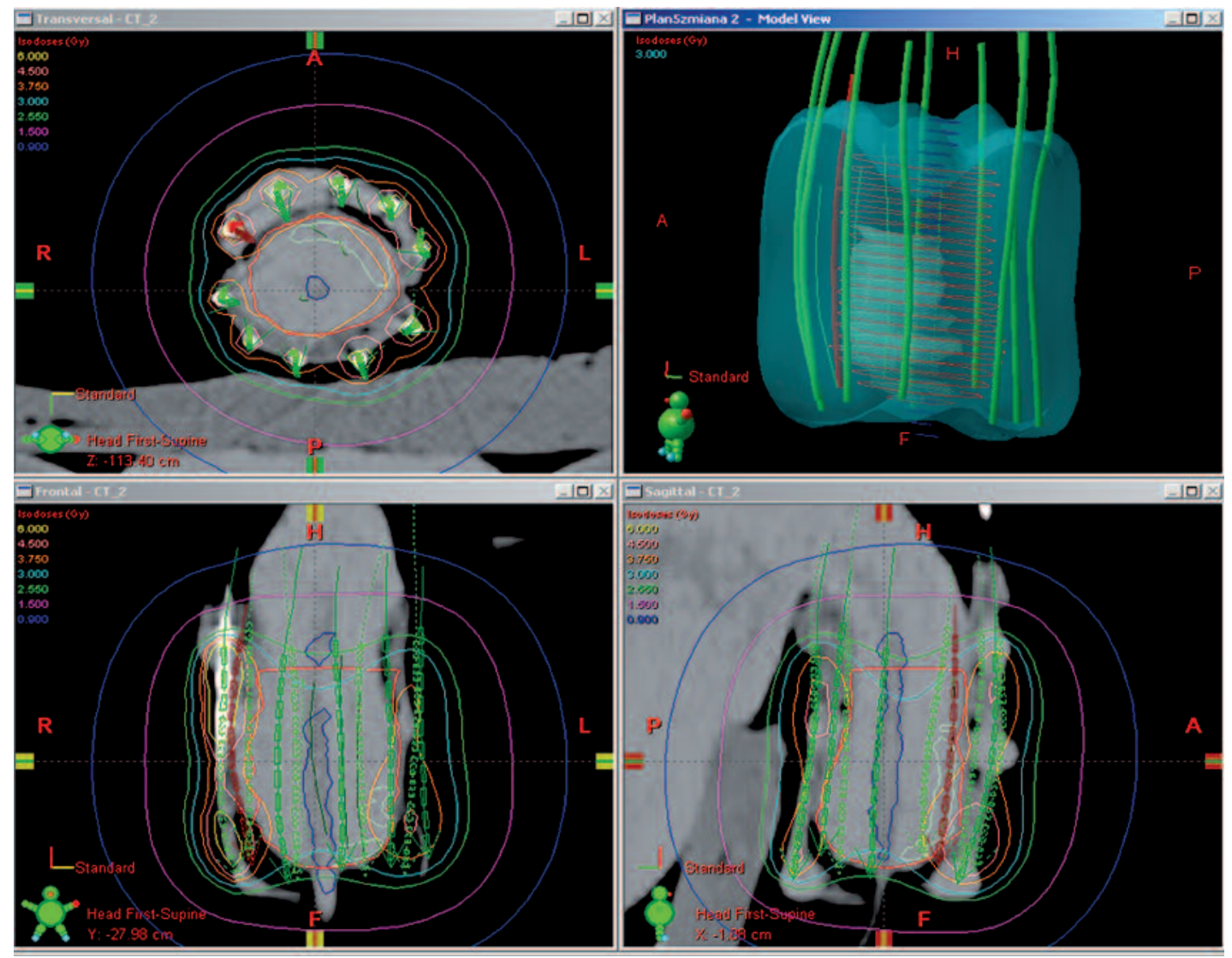

Fig. 11. III. Planning: CT based planning - patient with applicator in treatment position

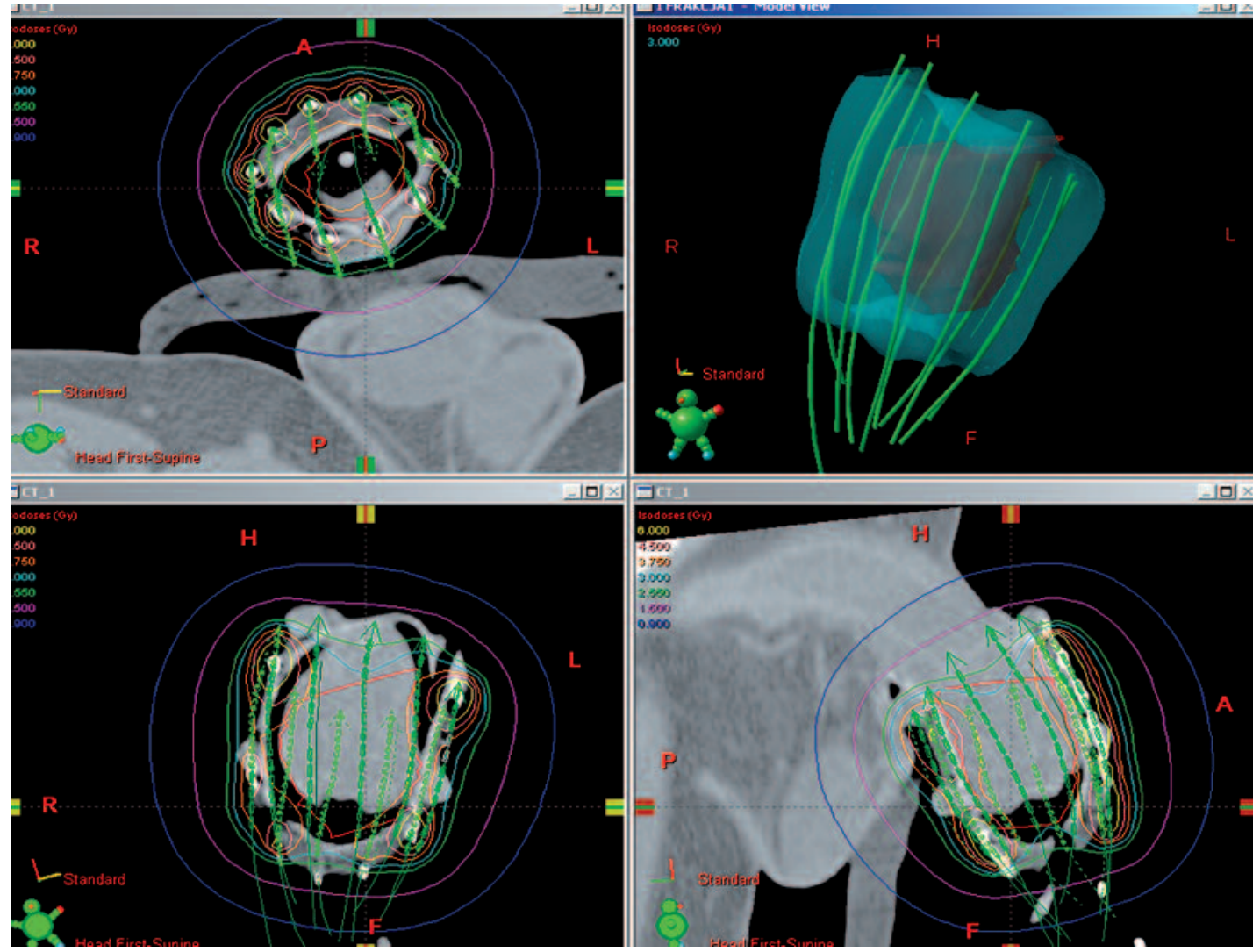

Fig. 12. III. Planning: CT based planning - patient with applicator in treatment position 


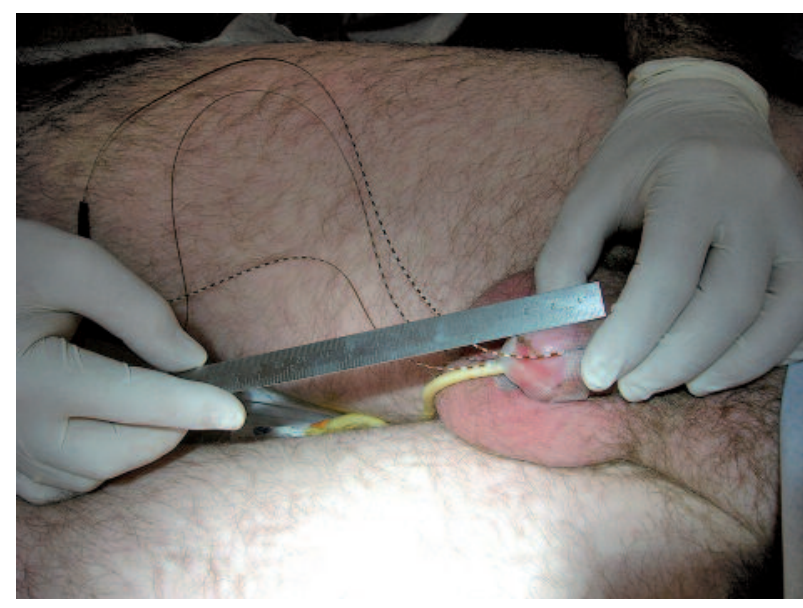

Fig. 15. IV. Therapy session: Preparing applicator, placing organ into it, Mosfet dosimetry, scrotum shielding, irradiation

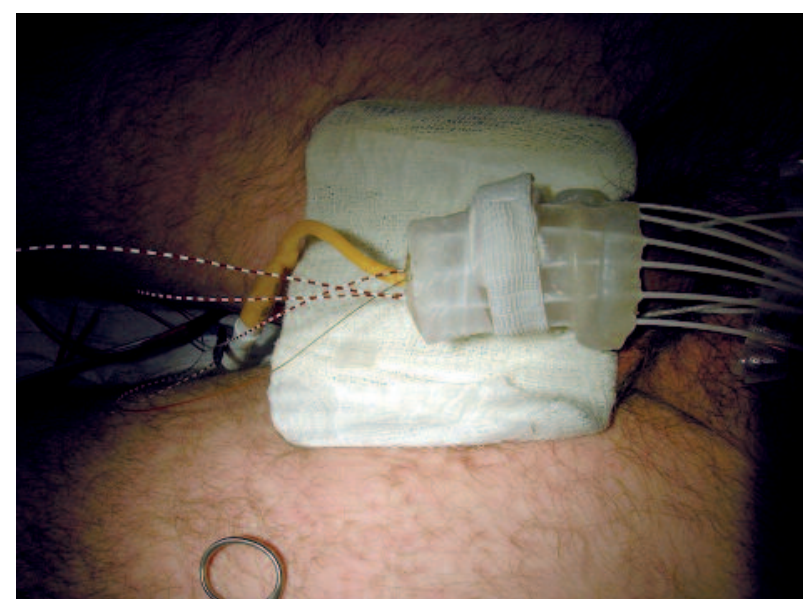

Fig. 17. IV. Therapy session: Preparing applicator, placing organ into it, Mosfet dosimetry, scrotum shielding, irradiation

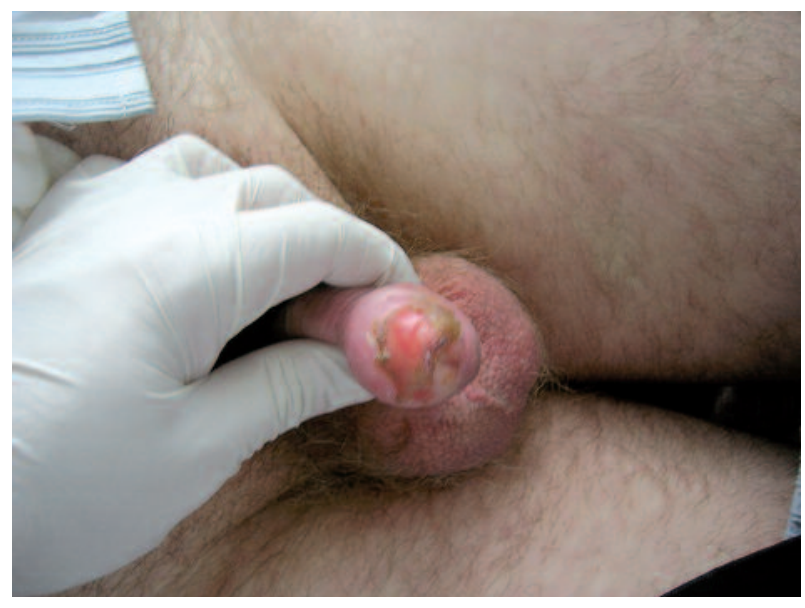

Fig. 19. V. Side effects: Target (penis) early reaction; Scrotum reaction

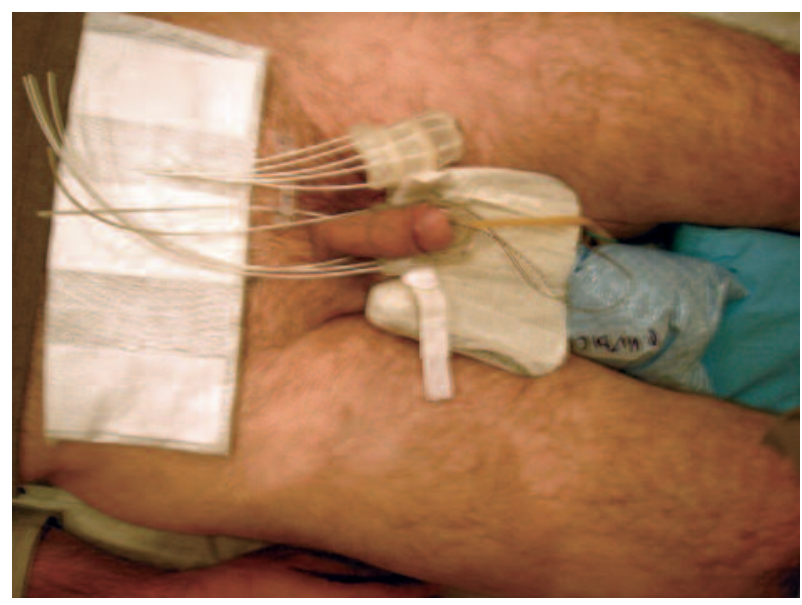

Fig. 16. IV. Therapy session: Preparing applicator, placing organ into it, Mosfet dosimetry, scrotum shielding, irradiation

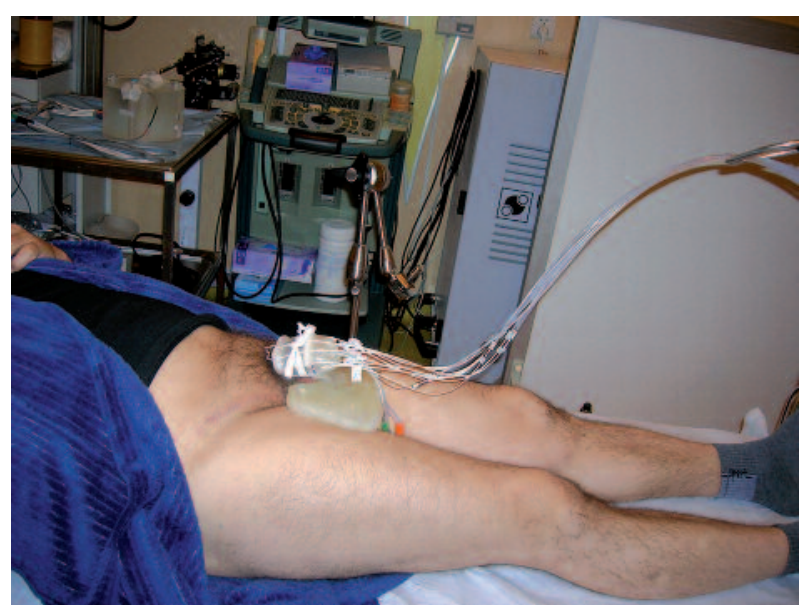

Fig. 18. IV. Therapy session: Preparing applicator, placing organ into it, Mosfet dosimetry, scrotum shielding, irradiation

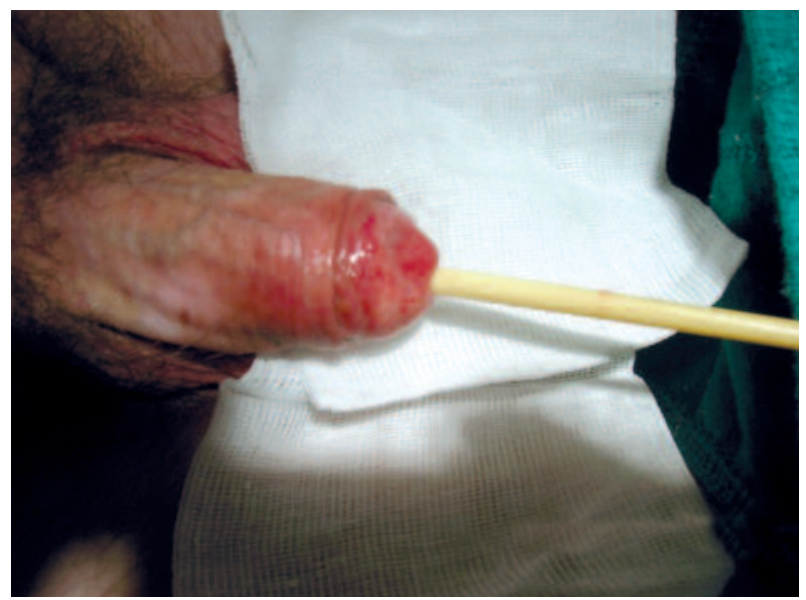

Fig. 20. V. Side effects: Target (penis) early reaction; Scrotum reaction 


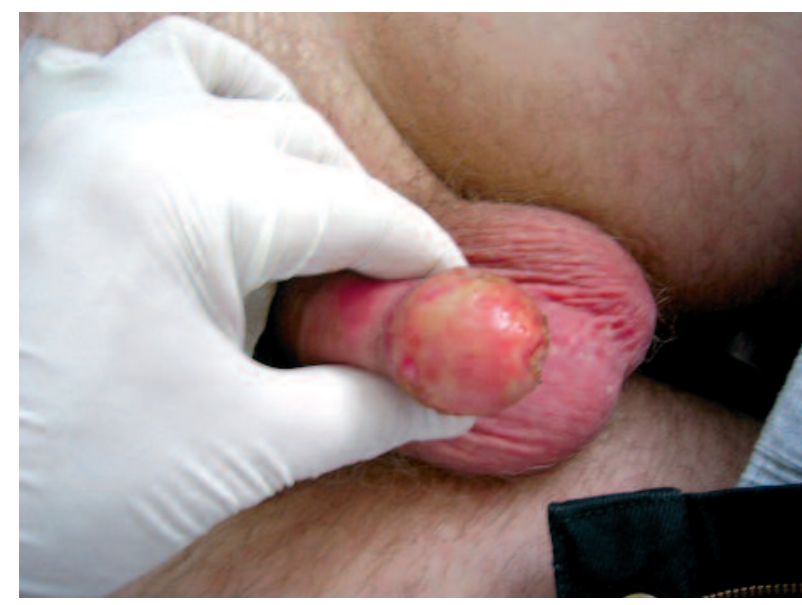

Fig. 21. V. Side effects: Target (penis) early reaction; Scrotum reaction

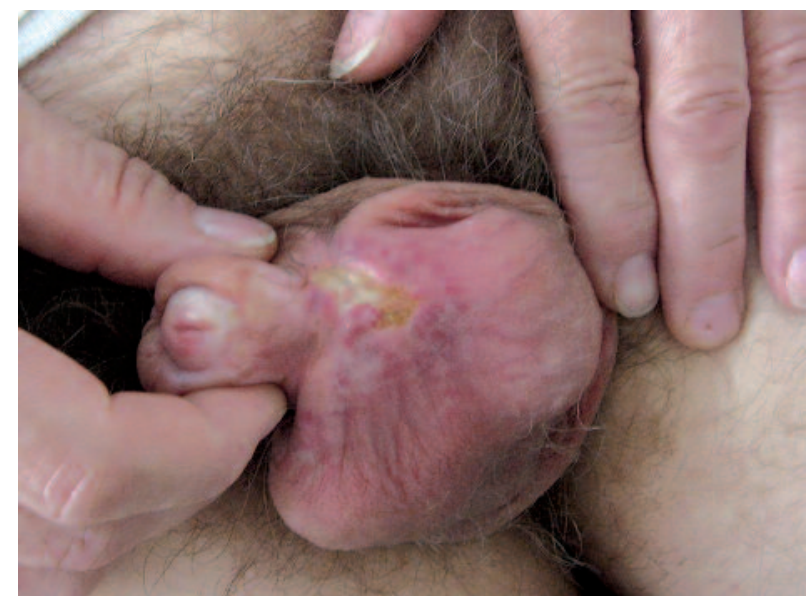

Fig. 23. V. Side effects: Target (penis) early reaction; Scrotum reaction

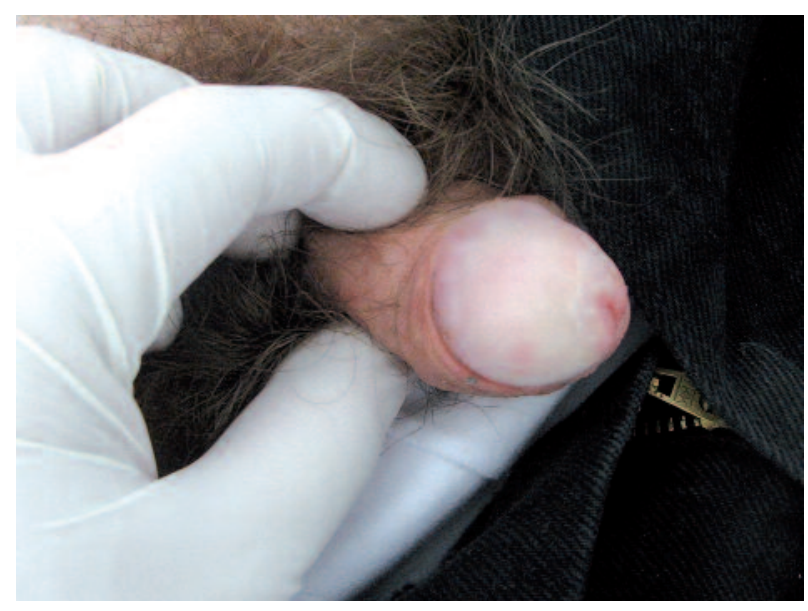

Fig. 25. VI. Results: Early visible effect

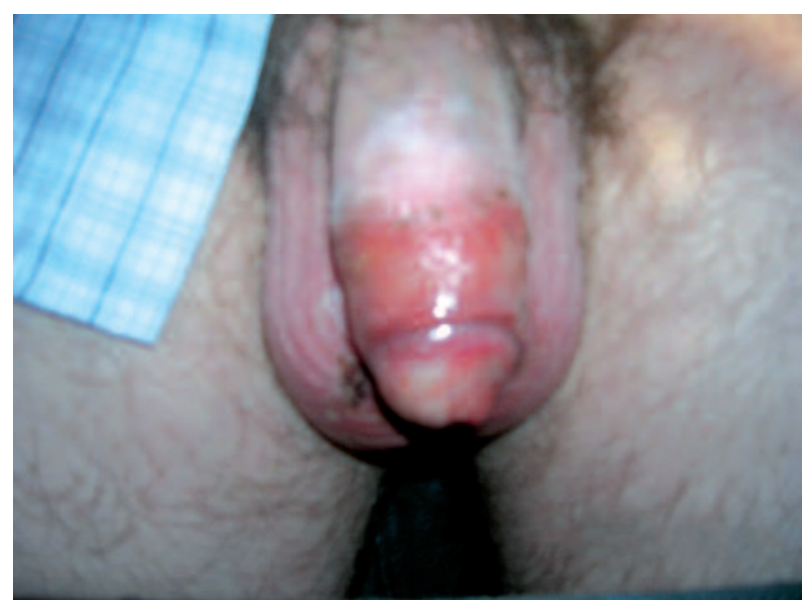

Fig. 22. V. Side effects: Target (penis) early reaction; Scrotum reaction

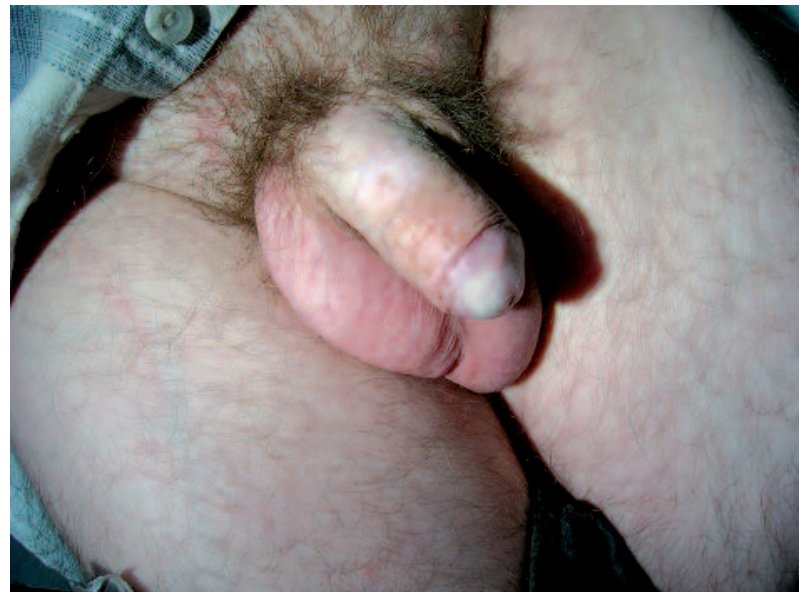

Fig. 24. VI. Results: Early visible effect 